\title{
An investigation of mass changes in the Bohai Sea observed by GRACE
}

\author{
Dapeng $\mathrm{Mu}^{1,2} \cdot$ Tianhe $\mathrm{Xu}^{1}\left[\right.$ [D Guochang $\mathrm{Xu}^{1}$
}

Received: 10 September 2019 / Accepted: 28 July 2020 / Published online: 8 August 2020

(c) The Author(s) 2020

\begin{abstract}
The Gravity Recovery and Climate Experiment (GRACE) satellite mission has profoundly advanced our knowledge of contemporary sea level change. Owing to the coarse spatial resolution and leakage issue across the land-ocean boundary, it is challenging (even impossible) for GRACE to detect mass changes over a region smaller than its spatial resolution, especially a semi-enclosed basin (e.g., the Bohai Sea) that is adjacent to land with significant mass variation. In this contribution, the causes for the GRACE RL06 mass changes in the Bohai Sea are investigated using a reconstruction technique that is implemented with multisource data, including altimeter observations, steric estimates, and land mass changes from GRACE RL06 mascon solution. Our results by the reconstruction technique demonstrate that the GRACE annual cycles are primarily caused by water mass changes rather than sediment changes. On the other hand, the mass trends from both reconstructed signals and those observed by the GRACE RL06 spherical harmonic coefficients (SHCs) are small, ranging from $-0.38 \mathrm{~mm} /$ year to $0.51 \mathrm{~mm} /$ year (depending on different data sources). Given that our estimated accuracies are $>0.8 \mathrm{~mm} /$ year (the real accuracies should be larger), our reconstructed results cannot directly confirm the presence of sediment accumulation or water mass increase; however, analysis of only the altimetry data suggests the mass trends are due to water mass increase, which would amount to $\sim 0.44 \mathrm{Gt} /$ year. Further investigation suggests that the mass trends in the Bohai Sea suffer from a $-2.9 \mathrm{~mm} /$ year leakage-in effect from groundwater depletion in the North China and about $2.5 \mathrm{~mm} /$ year signal attenuation (resulting in a $\sim 2.5 \mathrm{~mm} /$ year remaining trend that is roughly equivalent to the leakage-in trend, consequently leading to the small mass trend in the Bohai Sea). Our reconstruction results exemplify that elaborate data processing is necessary for specific cases. We also test whether the recently released RL06 mascon solutions that are resolved with constraints and require no further processing would improve the agreement with altimeter observations. We find that the seasonal cycles are improved relative to the RL06 SHCs; however, the rates derived from the mascon solutions cannot properly represent the altimeter-derived ocean mass estimates for the Bohai Sea, probably because the mascon solutions underestimate the rates or contain some processing artifacts. Nevertheless, the mascon solutions show enhanced signals, which offer new opportunities to investigate regional sea level change.
\end{abstract}

Keywords GRACE · Sea level change · Mass trend · The Bohai Sea

\section{Introduction}

Tianhe Xu

thxu@sdu.edu.cn

1 Institute of Space Sciences, Shandong University, Weihai, China

2 State Key Laboratory of Geodesy and Earth's Dynamics, Innovation Academy for Precision Measurement Science and Technology, Chinese Academy of Sciences, Wuhan, China
The causes for global mean sea level (GMSL) change can be mainly partitioned into three components (e.g., Stammer et al. 2013): (1) the steric contribution owing to temperature and salinity changes in seawater; (2) the mass addition from land water storage change and ice melting; (3) as well as the ongoing glacial isostatic adjustment (GIA) process (the response of the solid Earth to the past land-ocean mass exchange). The GIA effect can be modeled with the loading theory proposed by Farrell and Clark (1976). This theory was then improved by a vast body of literatures (e.g., Spada 2016 and the references therein). It was emphasized that the 
uncertainty associated with GIA correction was significant at global and regional scales (Tamisiea 2011; Uebbing et al. 2019). For instance, Chambers et al. (2010) showed that different GIA processing strategies could result in $\sim 1 \mathrm{~mm} /$ year difference maximum for global ocean mass trend. On the other hand, the steric component can be estimated from ocean models or reanalysis that assimilated various observations. However, understandings of the ocean mass changes remain in their nascency, at least before the twenty-first century.

The advent of Gravity Recovery and Climate Experiment (GRACE) satellite mission (Tapley et al. 2019), which was launched at March 2002, made it possible to directly investigate global/regional ocean mass variations. Since 2004, plentiful science reports related to ocean mass or sea level were based upon GRACE data alone (e.g., Chambers et al. 2004), or in conjunction with altimeter observations and Argo floats (e.g., Cazenave et al. 2009; Yi et al. 2015; Chambers et al. 2017; Cazenave et al. 2018). These results significantly improved our understandings of current climate change. For example, recent studies concluded the closure of GMSL budget by combining GRACE along with satellite altimeter and Argo floats (e.g., Chambers et al. 2017; Dieng et al. 2017). GRACE data indicated that more than $60 \%$ of the GMSL rise for the period 2003-2016 were attributed to ocean mass (Chen et al. 2018), indicating the dominant contribution of increased ocean mass to sea level rise. What is more, GRACE is capable of determining the role of different causes for GMSL rise in current climate (e.g., Rietbroek et al. 2016). For instance, overall GRACE estimates showed water mass gain in global land (excluding the area covered with glaciers and ice cap), consequently slowing the GMSL rise (by $0.71 \mathrm{~mm} /$ year for period 2002-2014; Reager et al. 2016).

The causes for regional sea level change, which differ substantially from those for GMSL, can also be interpreted with the help of GRACE estimates. For example, Wahr et al. (2014) studied seasonal variations of sea level in the Red Sea and found that mass contributions were larger than steric contributions. This finding was further confirmed by Feng et al. (2014) who used both GRACE data and ocean bottom pressure (OBP) records to investigate the mass variations in the Red Sea. Using an inversion method with GRACE data, Kusche et al. (2016) separated the mass and steric trends from altimetric sea level trend in the Bay of Bengal. They found that the mass trend (1.17-1.29 mm/year) was lower than the steric trend (4.3-4.6 mm/year).

Those valuable applications mentioned above, however, were implemented over large basins due to GRACE coarse resolution, which is commonly acknowledged to be $>300 \mathrm{~km}$ (Watkins et al. 2015). This leads us to a major question: Can GRACE measure mass changes in a small region (say $300 \mathrm{~km} \times 300 \mathrm{~km}$, or even smaller), especially over the coastal zone? The major challenges include the leakage and the stripes. By nature, the leakage results mainly from the coarse spatial resolution. For example, consider a mass variation block that is placed across the land-ocean interface. The signal in the oceanic part is typically contaminated by the signal in the land part (Watkins et al. 2015), because the land signals are usually one order of magnitude stronger than the oceanic signals, and they therefore leak into the oceans (Mu et al. 2017). Traditional correction method only reduces the leakage from the land into the oceans (e.g., Wahr et al. 1998), but fails to recover the oceanic signals over the coastal zone, whose signals are then masked out (e.g., Johnson and Chambers 2013). On the other hand, the spatial patterns of GRACE ocean mass change are shown to be distorted by the stripes, which originate from poor observability of east-west gradients (Watkins et al. 2015). The stripes compromise the variability, particularly in a small region. Although the stripes can be reduced by using the de-striping algorithms (e.g., Swenson et al. 2008), the real signals may be attenuated or even removed along with the stripes (Watkins et al. 2015). In summary, both the leakage and the stripes impede the understandings of mass changes in the coastal zone.

Additionally, other factors influencing coastal sea level change include sediment accumulations (e.g., Mouyen et al. 2018; sediment may replace the seawater, see Figure 3 from Liu et al. 2016), and subsidence associated with physical processes or human activities. For example, Liu et al. (2016) derived a $6 \mathrm{~mm} /$ year rate of sediment accumulation for the East China Sea by using GRACE data with consideration of leakage reduction. This rate was in agreement with the sediment-core measurements. Using multisource data including GRACE estimates, Chang et al. (2019) confirmed the finding of the trends by Liu et al. (2016). More importantly, Chang et al. (2019) identified significant seasonal changes in sediment, which were driven by ocean current between the East China Sea and the Yellow Sea.

In this contribution, we make use of multisource data, including various GRACE observations (the latest RL06 version), to explore the causes for the mass changes in the Bohai Sea, a small and semi-enclosed basin. By reconstructing GRACE-type signals using multisource data, we find that the annual cycles of the water mass changes in the Bohai Sea are mainly induced by water mass variations rather than sediment accumulation. We acknowledge that, at this point, it is not conclusive to determine the cause for the mass trends due to high uncertainty. The mascon solutions are compared to the traditional spherical harmonic coefficients (SHCs). Their merits and limitations are both discussed. 


\section{Materials and methods}

\subsection{Study area}

The Bohai Sea is one of China's seas, which is surrounded by three provinces (Shandong, Liaoning and Hebei) and Tianjin City (Fig. 1); it covers an area of $\sim 80,000 \mathrm{~km}^{2}$ with mean depth of $\sim 18 \mathrm{~m}$ and maximum depth of $\sim 80 \mathrm{~m} \mathrm{(Bi}$ et al. 2015). As a semi-enclosed shallow-water basin, the Bohai Sea provides valuable recourse (e.g., fishery and oil) for regional economy system and even affects national development. Sea level changes in the Bohai Sea have a significant impact on regional climate and human activities. It is therefore of importance to investigate the causes for the sea level change in the Bohai Sea.

When we aim to explore the causes for the Bohai Sea level change, we must consider the effect of sediment. Indeed, the Yellow River (Fig. 1), which ranks second in the world in terms of sediment load (e.g., Bian et al. 2013), transports about $1 \mathrm{Gt} /$ year sediment into the Bohai Sea. Some studies argued that only 10-30\% of the sediment was carried out of the Bohai Sea, resulting in an accumulation in the Bohai Sea itself (Note that the time scale was across 7000 years; Yang and Liu 2007). As the Bohai Sea does experience sediment accumulation, a key question is whether GRACE can see the sediment accumulation, or just the water mass change, or both? To answer these questions, we use various GRACE estimates along with altimeter observations and other datasets.

\subsection{Altimeter observations}

We use sea surface height (SSH) anomalies provided by the Archiving, Validation, and Interpretation of Satellite

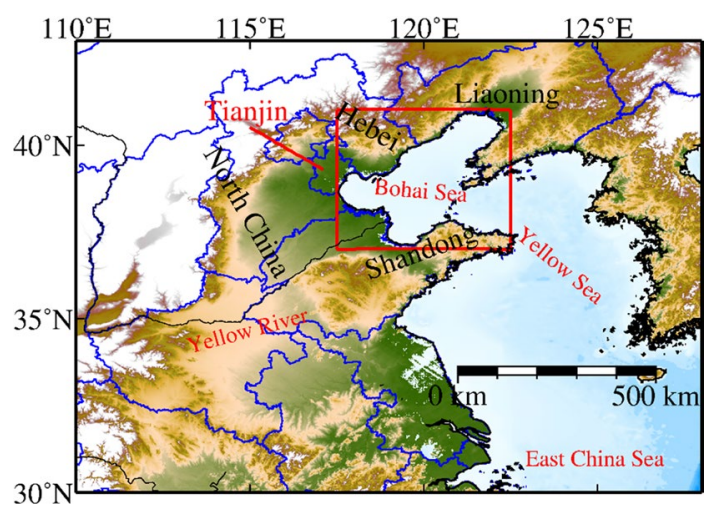

Fig. 1 Map of the Bohai Sea (i.e., oceanic area enclosed in red box); Shandong, Liaoning and Hebei are three provinces in China, and Tianjin is one of municipalities directly under the central government. The area of the Bohai Sea is about $80,000 \mathrm{~km}^{2}$
Oceanographic (AVISO) data processing center, covering the period 2002-2014. The AVISO data merged several altimeter observations, e.g., T/P, Jason-1/2 and Envisat. All standard corrections (including inverted barometer effect) had been done (AVISO 2014). The monthly AVISO data were gridded into $0.25^{\circ} \times 0.25^{\circ}$ horizontal resolution.

\subsection{GRACE data}

We use two types of GRACE data, including the classic SHCs (i.e., the Level-2 data) and the mascon solutions (Watkins et al. 2015; Save et al. 2016). Both are the latest released version, i.e., RL06.

The version RL06 of SHCs generated by three centers [the Center for Space Research (CSR), the GeoForschungsZentrum (GFZ), and the JPL] are used in this paper. Before these SHCs are converted into mass variations in terms of equivalent water height (EWH), several standard procedures are necessary. We include degree one coefficients computed by Swenson et al. (2008) into each SHCs. We replace the GRACE $\mathrm{C}_{20}$ coefficients with those resolved with satellite laser ranging (Cheng et al. 2013a). To obtain the ocean mass changes, the atmospheric and oceanic mass (i.e., the so-called GAD product; Flechtner et al. 2014) are added back, and the surface atmospheric mass changes are removed following the recommendation by Uebbing et al. (2019). We convert the corrected SHCs into mass variations using $300 \mathrm{~km}$ Gaussian filter (e.g., Wahr et al. 1998; Guo et al. 2014; Yan et al. 2016). Finally, the GIA effect estimated by the model ICE-6G-D (Peltier et al. 2017) was removed from the mass variations. The GIA correction ranges around $0.4 \mathrm{~mm} /$ year for the Bohai Sea, which has significant contribution to GRACE estimates. While the Gaussian filter is useful to reduce the noise in GRACE estimates, it causes spatial leakage, especially at the land-ocean boundary like the Bohai Sea. Instead of correcting the leakage, we here employ a reconstruction technique to reproduce GRACEtype signals as much as possible (see Sect. 3.2).

The second type of GRACE estimates, i.e., the mascon solutions, included the JPL RL06 mascon (version 02) (Watkins et al. 2015) and CSR RL06 mascon (version 01) (Save et al. 2016). Hereinafter, those two mascon are referred to as JPL-M and CSR-M, respectively. Readers can find more context about mascon concept from Rowlands et al. (2010). In short, mascon represent directly the global surface mass flux, including changes in OBP. Technically, these two mascon series were resolved with different approaches and spatial resolutions. For instance, JPL applied model constraints (the constraints were constructed with changes in OBP from ocean model, i.e., external information) to their mascon, whereas CSR designed time-variable regularization matrix for their mascon using purely GRACE information. JPL resolved their mascon at $3^{\circ} \times 3^{\circ}$ block, but CSR generated 
their mascon with a higher grid resolution of $\sim 120 \mathrm{~km}$. Users should be aware of that the $120 \mathrm{~km}$ is only a grid resolution, it does not represent a real spatial resolution. The GIA effect has been corrected for these two mascon solutions, which both used the model ICE-6G-D (Peltier et al. 2017). It is noteworthy that CSR interpolated their original grid to a regular $0.25^{\circ}$ grid.

As mentioned above, a major issue in recovering the coastal ocean mass is the leakage. JPL and CSR used different methods to deal with this issue. We first describe the method used by JPL. Consider a $3^{\circ} \times 3^{\circ}$ block lying across both land and oceans. In theory, this block contains signals that are mixed with land mass and ocean mass, but we cannot discriminate the ocean mass from this block mass variation unless additional information is available. JPL developed a CRI algorithm (Wiese et al. 2016) to separate the land mass and ocean mass from the coastal block mass variations. The CRI algorithm used information from adjacent mascon block mass variations to determine the coastal ocean/land mass variations under the principle of conserving the mass. On the other hand, CSR mascon reduced signal leakage into oceans using their designed regularization matrix (i.e., the leakage correction is done during data generation).

\subsection{Steric estimates}

We use the EN4 data produced by Met Office Hadley Centre (Good et al. 2013) to estimate the steric change in the Bohai Sea. The EN4 data were generated with objective analysis of a global quality controlled ocean temperature and salinity profiles, including WOD09 dataset and Argo floats; it provides users a grid with $1^{\circ}$ spatial resolution in the horizontal (covering global major basins, including coastal zone) and 42 unevenly spaced levels in the vertical (from the surface to $5000 \mathrm{~m}$ depth). We calculate the steric change using the method by Llovel and Lee (2015).

It is worth mentioning that the profiles used by EN4 are dramatically sparse over China seas, including the Bohai Sea, implying that the steric estimates for the Bohai Sea was by method rather than real observations. Some studies argue that EN4 had reduced variability than the ocean reanalysis owing to lacking knowledge of dynamics (e.g., geostrophy and wave dynamics, e.g., Carton et al. 2019). We thus examine an ocean reanalysis, namely the Ocean ReAnalysis System 5 (ORAS5; Zuo et al. 2019), to identify any significant bias in the EN4 data. The ORAS5 has the same spatial horizontal resolution as EN4 but with 75 uneven vertical levels. It is worth mentioning that the ORAS5 employed the same hydrography as EN4, additionally, it contained ocean dynamics (e.g., the ORAS5 was forced with Europe Center for Medium Range Weather Forecasts atmospheric reanalysis interim).
Both EN4 and ORAS5 suggest evident seasonal cycles that dominate the steric sea level change in the Bohai Sea (Fig. 2). While the EN4 estimates are generally consistent with the ORAS5 estimates, their differences are noticeable. We derive a $\sim 8 \mathrm{~mm}$ root mean squares for their differences. Nevertheless, our steric estimates rely on the EN4 data.

\section{Results}

In this section, we first analyze the GRACE mass trends from SHCs, then use multisource data to reconstruct the gravity change observed by GRACE. The reconstruction allows us to interpret the gravity change physically, which consequently helps to determine the causes. Finally, we investigate the mass changes in the Bohai Sea using GRACE mascon.

\subsection{GRACE mass trends from SHCs}

We compute the linear trends (along with annual and semiannual cycles) for GRACE mass changes derived from SHCs, which are smoothed with $300 \mathrm{~km}$ Gaussian filter (Wahr et al. 1998; Guo et al. 2014). The mass changes are expressed in terms of EWHs (the first row in Fig. 3). The most evident feature in the trends pattern is the negative rate in the North China Plain, which were induced by groundwater depletion. This groundwater mass loss, which can be derived by removing the surface soil moisture change from GRACE observations, was estimated to be $7.2 \pm 1.1 \mathrm{Gt} /$ year during 2002-2014 (Feng et al. 2018). Another signal is the mass increase over the East China Sea. These positive rates are linked to sediment accumulation $(0.82 \pm 0.3 \mathrm{Gt} /$ year $)$ by Chang et al. (2019), who also concluded seasonal cycles for sediment change.

While the western Bohai Sea shows visible negative rates, positive rates exhibit over the eastern Bohai Sea, especially for CSR SHCs. Since the SHCs are smoothed with Gaussian filter that leads to leakage issue (the leakage causes signal

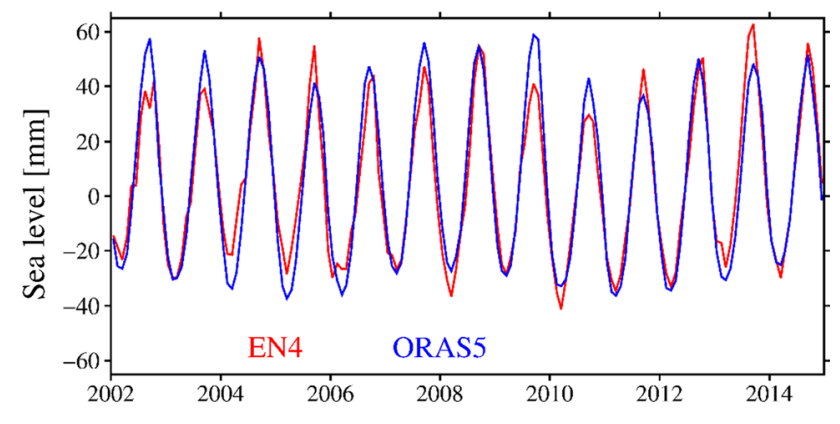

Fig. 2 The steric sea level change in the Bohai Sea. The red line is from EN4, and the blue line is from ORAS5 

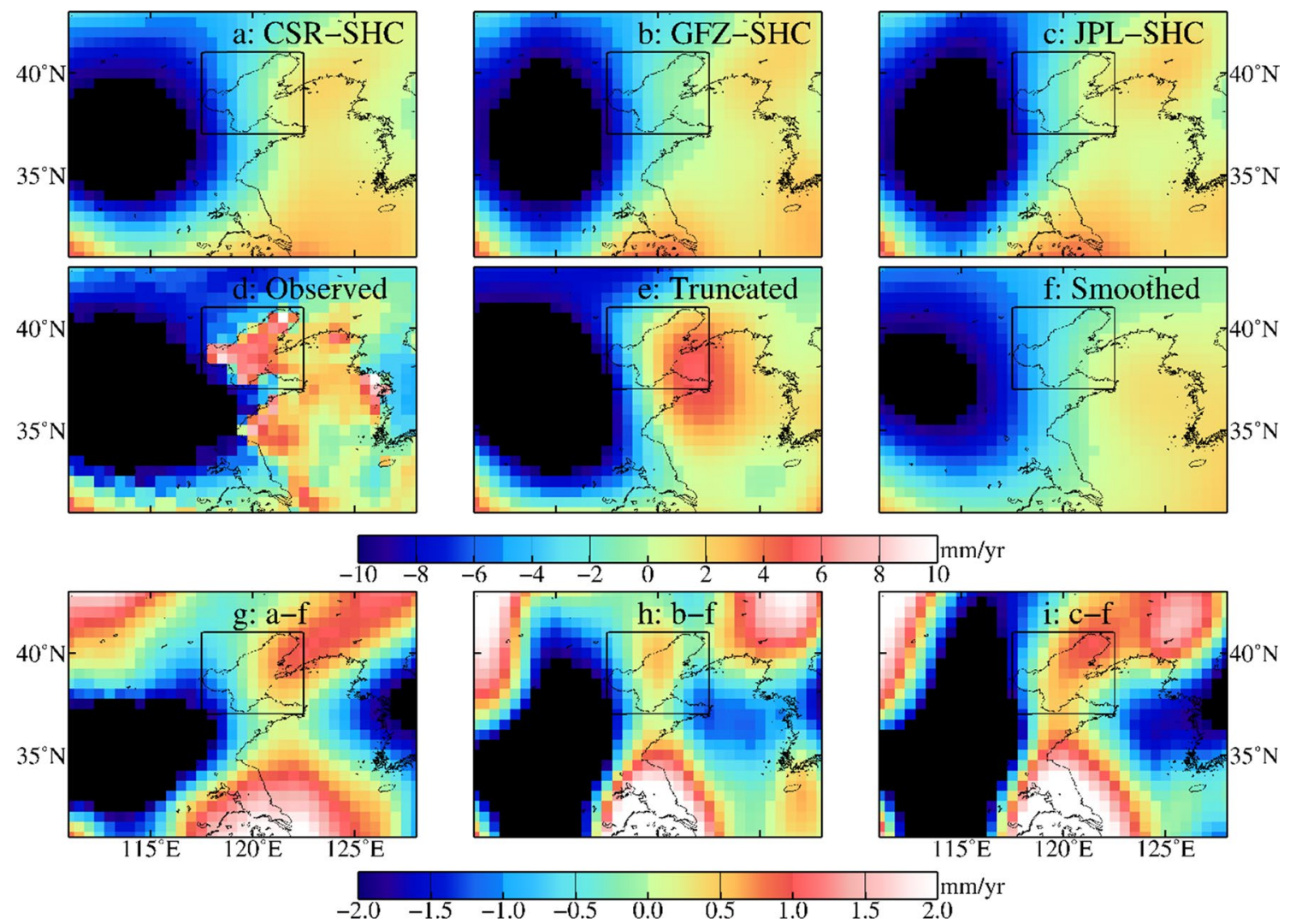

Fig. 3 Mass trends from GRACE SHCs and the reconstructed signals. The first row: GRACE mass trends from SHCs with $300 \mathrm{~km}$ Gaussian filter, a CSR; b GFZ; and c JPL. The significant mass loss in the North China was due to groundwater depletion (e.g., Feng et al. 2018). The second row: trend patterns from the reconstructed sig- nal, $\mathbf{d}$ the observed mode, note this mode is derived using the input data for the reconstruction (see Sect. 3.2); $\mathbf{e}$ the truncated mode; $\mathbf{f}$ the $300 \mathrm{~km}$ Gaussian smoothed mode. The third row $(\mathbf{g}-\mathbf{i})$ : the difference between the smoothed mode and CSR, GFZ, and JPL loss and disrupts its spatial pattern), it is therefore rational to speculate that these negative rates may be (partly) caused by the leakage from huge mass loss in the North China Plain. This raises two interesting questions: (1) what is the true spatial pattern of mass signal in the Bohai Sea and (2) what causes the mass changes in the Bohai Sea, water mass or sediment?

Before answering these two questions, it is helpful to examine the temporal evolution of mass changes in the Bohai Sea. In Fig. 4, we plot the time series of mass changes derived from 3 SHCs products. One prominent feature in these records is the seasonal cycle, associated with high frequency variability, though. We fit the annual and semiannual cycles, and a linear trend to the Bohai time series derived from SHCs. The resulting trends along with annual amplitudes are shown in Table 1. The differences in the mass changes derived from $3 \mathrm{SHCs}$ products are visible. For instance, JPL amplitude is found to be the smallest $(\sim 18 \%$ smaller than CSR amplitude). On the other hand, more discrepancies are shown for the trends. The CSR SHCs suggests a small positive trend, amounting to $0.16 \pm 0.63 \mathrm{~mm} /$ year, whereas the other two SHCs produce negative trends. Notably, the magnitude of JPL trend is much larger than the magnitude of the other two SHCs. These discrepancies indicate inherent uncertainty in GRACE estimates due to different data processing strategies.

To interpret the causes for the mass changes in the Bohai Sea, external information is needed. In the following section, using GRACE data along with other data sources, we investigate the causes for the mass change in the Bohai Sea with a reconstruction technique. We use several "observed" data to reconstruct GRACE-type signals, hence interpreting the causes.

\subsection{Reconstruction of GRACE-type signals}

In this section, we reconstruct the mass change in the Bohai Sea (Fig. 2) by using several datasets, including altimeter observations, steric estimates, and mass changes in the North China Plain observed by GRACE. We first describe the role of different datasets, then elaborate the 

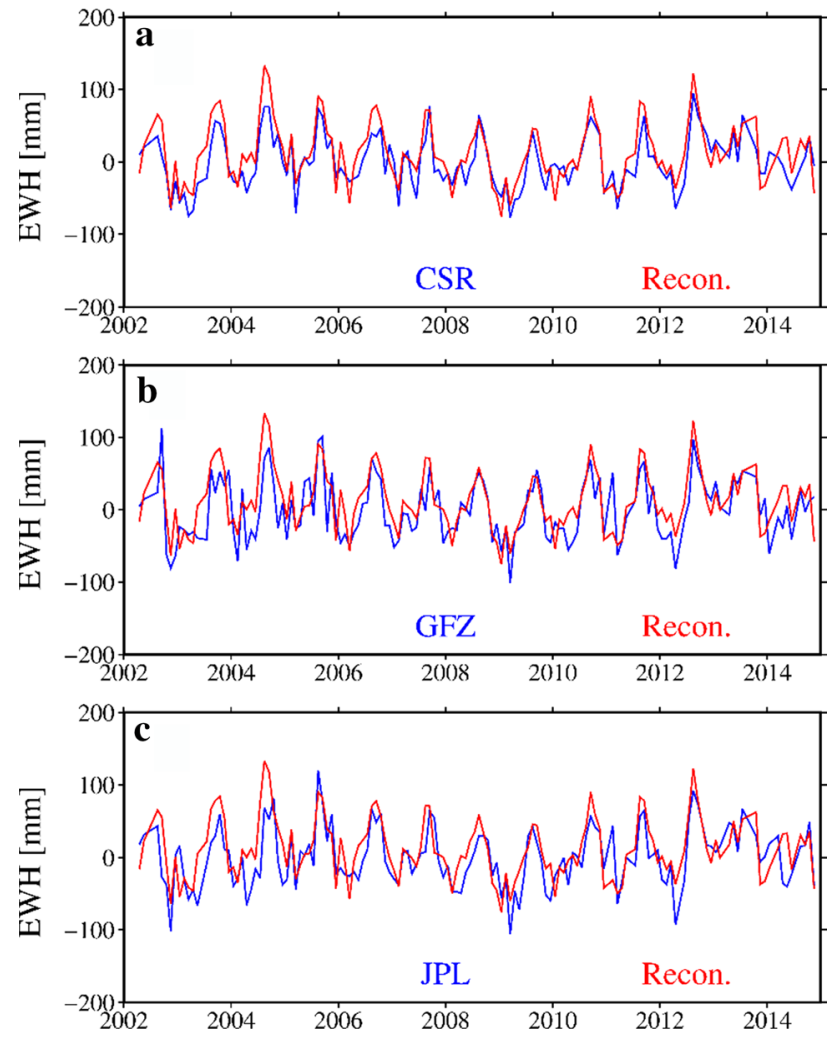

Fig. 4 EWH time series from different datasets. Note that the time series is a mean averaged over the box (oceanic parts only) defined in Fig. 1. The blue lines in panel (a-c) are from SHCs products released by CSR, GFZ, and JPL, respectively; and the red line is from our reconstruction (Sect. 3.2)

procedure for the reconstruction in details, which can be replicated by the readers.

In total, three kinds of datasets are used for the reconstruction:

1. AVISO This dataset contains the SSH measured by altimeters. After being corrected for the steric height, the SSH represents the changes in local geometrical height, which may be caused by seawater increase or sediment accumulation, or both.
2. EN4 This dataset provides the temperature and salinity of seawater in different depths. We calculate the steric height change using EN4, then remove the steric height from the AVISO SSH.

3. CSR-M This dataset serves to represent the mass changes in land due to its highly resolved resolution. The CSR-M is used to evaluate the leakage-in effect on mass change in the Bohai Sea.

We illustrate the data processing for the reconstruction of GRACE-type signals. First, the grids resolution of each dataset should be consistent. The EN4 data are interpolated on the grid that are the same as those of AVISO (i.e., $0.25^{\circ} \times 0.25^{\circ}$, this interpolation will be convenient for the following calculations). Second, we subtract the steric height from the AVISO SSH, and then convert the resulting AVISO SSH, and CSR-M mass change into SHCs up to degree 60. Finally, these SHCs are converted back to the spatial domain (i.e., EWHs) with $300 \mathrm{~km}$ Gaussian filter (Wahr et al. 1998; Yan et al. 2016), which agrees with the SHCs data processing.

Our key issue here is to determine whether the Bohai Sea experiences sediment accumulation or water mass change. We assume that the AVISO SSH are caused by water mass change. (This means that the geometrical water height equals to EWH.) Given the density of sediment is larger than the density of water $\left(1.6 \mathrm{~g} / \mathrm{cm}^{3}\right.$ vs. $\left.1.0 \mathrm{~g} / \mathrm{cm}^{3}\right)$, the reconstructed mass trends should be smaller than the GRACE mass trends if sediment accumulation occurs at the Bohai Sea. Otherwise, there is no sediment accumulation, or it is not significantly detectable by GRACE.

In the second row of Fig. 3, we show the input data, which are called the "observed" mode. The input data are converted into SHCs and truncated at degree 60 (Fig. 3e) and then smoothed with $300 \mathrm{~km}$ Gaussian filter (Fig. 3f). To further evaluate the reconstruction, we average the (oceanic) grids defined in the box shown in Fig. 3 or Fig. 1 for the smoothed mode (Fig. 3f). The resulting time series, along with those from SHCs are displayed in Fig. 4.

By eye, we can see that the reconstructed mass changes agree with those from SHCs (Fig. 4), especially the CSR SHCs, which suggest the highest correlation
Table 1 Rates and annual amplitudes derived from GRACE SHCs and the reconstructed signals

\begin{tabular}{lllllll}
\hline Data & CSR & GFZ & JPL & Recon. & Recon-1 & Recon-2 \\
\hline Rate (mm/year) & $0.21 \pm 0.85$ & $-0.17 \pm 0.91$ & $0.51 \pm 0.94$ & $-0.38 \pm 0.82$ & $-2.91 \pm 0.51$ & $2.53 \pm 0.43$ \\
Amplitude (mm) & $36 \pm 14$ & $38 \pm 14$ & $34 \pm 14$ & $45 \pm 17$ & $11 \pm 4$ & $34 \pm 8$ \\
Phase (day) & $350 \pm 40$ & $340 \pm 40$ & $350 \pm 45$ & $330 \pm 30$ & $355 \pm 45$ & $320 \pm 30$ \\
RMS (mm) & 27 & 29 & 29 & 26 & 19 & 17 \\
\hline
\end{tabular}

RMS = root mean squares of the residuals that have the linear trend and seasonal cycles removed; Recon. $=$ Reconstruction, which means that multisource data are used to "reconstruct" GRACE-type signals (see Sect. 3.2). Note that recon-1 uses only GRACE land mass, and recon-2 uses only steric-corrected altimeter observations 
$(R=0.85 \pm 0.05)$. The GFZ SHCs and JPL SHCs produce correlations of $0.75 \pm 0.06$ and $0.73 \pm 0.06$, respectively. From Fig. 4a, it is observed that the mass changes of both the reconstructed signal and CSR SHCs is dominated by the nonlinear mass variability (i.e., detrended mass changes, including annual cycles and interannual variability). From Table 1, it is clear that a good agreement of annual cycles is shown between the reconstructed signal and the CSR SHCs; it is confident to attribute the annual cycles of the mass changes in the Bohai Sea to water mass changes. If the annual cycles are removed from the reconstructed signal (or the CSR SHCs), the residuals are derived, mainly including the interannual variability and high frequency ( $<6$ months) variability. We find that the correlation between two residuals (the reconstructed signals and CSR SHCs) drops to $0.61 \pm 0.12$. In the meanwhile, we observe no obvious unusual anomalies in those residuals.

The reconstructed trend is small and close to the trend from GFZ SHCs (Table 1). However, the sign of the reconstructed trend is in opposite to those of CSR and JPL SHCs. These small trends pose a challenge to unraveling the cause for the mass trends in the Bohai Sea, given that our estimated uncertainty in GRACE mass variations is larger than these mass trends. Additionally, our uncertainty is derived from the formal error of least squares fit, which means that the uncertainty is likely underestimated (for instance, a different GIA model (e.g., the GIA model by A et al. (2013) may yield a different correction up to $\sim 1 \mathrm{~mm} /$ year for the Bohai Sea). Therefore, it is not conclusive to attribute the mass trend to water mass increase or sediment accumulation.

Although we lack enough evidences to claim the water mass increase or reject the sediment accumulation in the Bohai Sea, the altimetry measurements appear to suggest an increase in water mass. If this water mass increase holds, we can calculate the "true" mass increase from the "observed" mode (Fig. 3d). The steric-corrected altimeter measurements indicate that the Bohai Sea has an evident mass trend of $5.23 \pm 1.14 \mathrm{~mm} /$ year (see Table 2). Totally, this mass trend amounts to $0.44 \pm 0.08 \mathrm{Gt} /$ year water mass change in the Bohai Sea.

We find that the smoothed mode (Fig. 3f) is somewhat different to the GRACE SHCs pattern (The first row in
Fig. 3). To investigate this issue further, we compute the difference between the smoothed mode and the GRACE SHCs (The third row in Fig. 3). Spatially, the trend differences are visible over the Bohai Sea, and especially the North China and the Yellow Sea, hinting the uncertainties associated with both GRACE estimates, steric estimates (Fig. 1), and altimeter observations. To determine the cause for the mass trend in the Bohai Sea, future work should either reduce those uncertainties or present more evidence.

More insights can be gained if the leakage-in effect and signal attenuation are quantified, respectively. To do so, we carry out two reconstructions (Fig. 5). The first one uses CSR-M that include only land grids (referred to as "recon1 "), which simulates the leakage-in effect. The second reconstruction uses the steric-corrected altimeter observations (referred to as "recon-2"), allowing us to evaluate the signal attenuation due to smoothing. The resulting time series are shown in Fig. 5, and the statistical results are illustrated in Table 1 . The recon- 1 has a $-2.41 \pm 0.45 \mathrm{~mm} /$ year trend contribution to the mass changes in the Bohai Sea. This negative trend mainly originates from the mass loss in the North China. The mass changes in Liaoning have negligible contribution, because the recon-1 time series have almost not been changed if the grids in Liaoning are masked out. Compared to the original steric-corrected altimeter observations (i.e., the inferred mass changes, see Table 2),

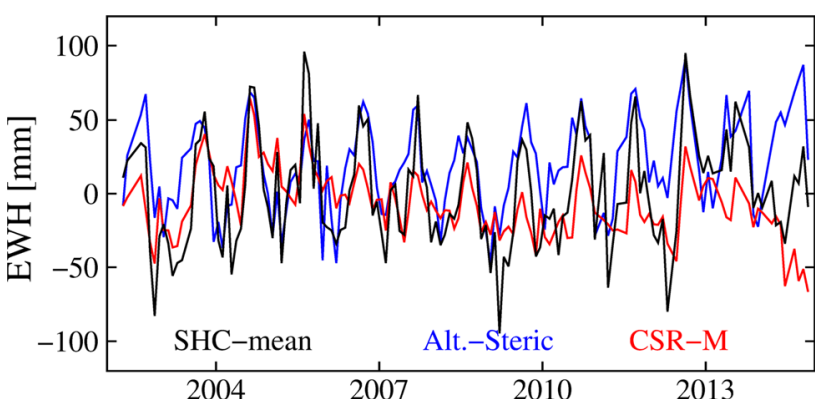

Fig. 5 Mass changes from GRACE SHCs and different reconstructed signals. The blue line and red line represent the reconstructed signal derived using steric-corrected altimeter observations and CSR-M (RL06) land mass, respectively, and the black line represents the mean of 3 GRACE SHCs
Table 2 Rates and annual amplitudes derived from GRACE mascon, the inferred mass changes, along with altimeter observations and steric estimates

\begin{tabular}{llllllll}
\hline Data & AVISO & SUM-1 & SUM-2 & EN4 & Inferred & JPL-M & CSR-M \\
\hline Rate (mm/year) & $6.12 \pm 1.35$ & $7.28 \pm 0.96$ & $1.52 \pm 0.75$ & $0.93 \pm 0.33$ & $5.23 \pm 1.14$ & $6.34 \pm 0.96$ & $0.59 \pm 0.86$ \\
Amplitude (mm) & $128 \pm 19$ & $102 \pm 14$ & $108 \pm 12$ & $52 \pm 12$ & $97 \pm 11$ & $53 \pm 10$ & $67 \pm 10$ \\
Phase (day) & $320 \pm 25$ & $340 \pm 30$ & $330 \pm 30$ & $364 \pm 35$ & $300 \pm 35$ & $320 \pm 35$ & $310 \pm 13$ \\
RMS (mm) & 41 & 36 & 26 & 11 & 43 & 33 & 25 \\
\hline
\end{tabular}

SUM-1 is the combination of JPL-M CRI and EN4; SUM-2 is the combination of CSR-M and EN4; the inferred result is derived by subtracting the EN4 from AVISO. The definition of RMS is the same as in Table 1 
the recon-2 time series suggest that the signals in the Bohai Sea suffer a dramatically signal attenuation, leading to $50 \%$ signal loss. The remaining trend is almost compensated by the leakage-in trend, consequently resulting in a small mass signal (Table 1). These two reconstructions demonstrate that both leakage-in effect and signal attenuation have vital (basically equal) impact on the mass changes in the Bohai Sea (Fig. 6a, c).

\subsection{Mascon solutions}

GRACE mascon solutions provide some insights into sea level change in the Bohai Sea. We remove the steric estimates derived from EN4 from the AVISO SSH (This data processing does not involve any special techniques, e.g., filtering.) The resulting time series represent the mass changes, which are referred to as the "inferred" mass (i.e., the stericcorrected altimeter observations, which is comparable to the "observed" mode; Table 2). We combine the mascon solutions with the steric estimates, and refer to it as the "SUM" sea level (Fig. 7). To obtain the mean Bohai Sea level, we average the (oceanic) grids within the box defined in Fig. 1 . The amplitude of both JPL-M and CSR-M are found to be substantially smaller than the inferred amplitude (Table 2). The difference in the phase between the inferred mass and mascon solutions is also noticeable. It is not surprising that the SUM amplitude (both SUM-1 and -2) is smaller than the AVISO amplitude (Table 2), suggesting it is challenging to close the sea level budget on seasonal scale using mascon.

Surprisingly, the CSR-M rate is substantially smaller than the JPL-M rate (Table 2) that is estimated from the mascon with application of CRI algorithm. This difference may be partly caused by the treatment of leakage correction. To correct the leakage across the land-ocean interface, JPL-M adopted a postprocessing strategy, i.e., the CRI algorithm, whereas CSR-M used a direct approach during the mascon generation to prevent the leakage (Save et al. 2016). The difference between the CSR-M and the inferred trend implies
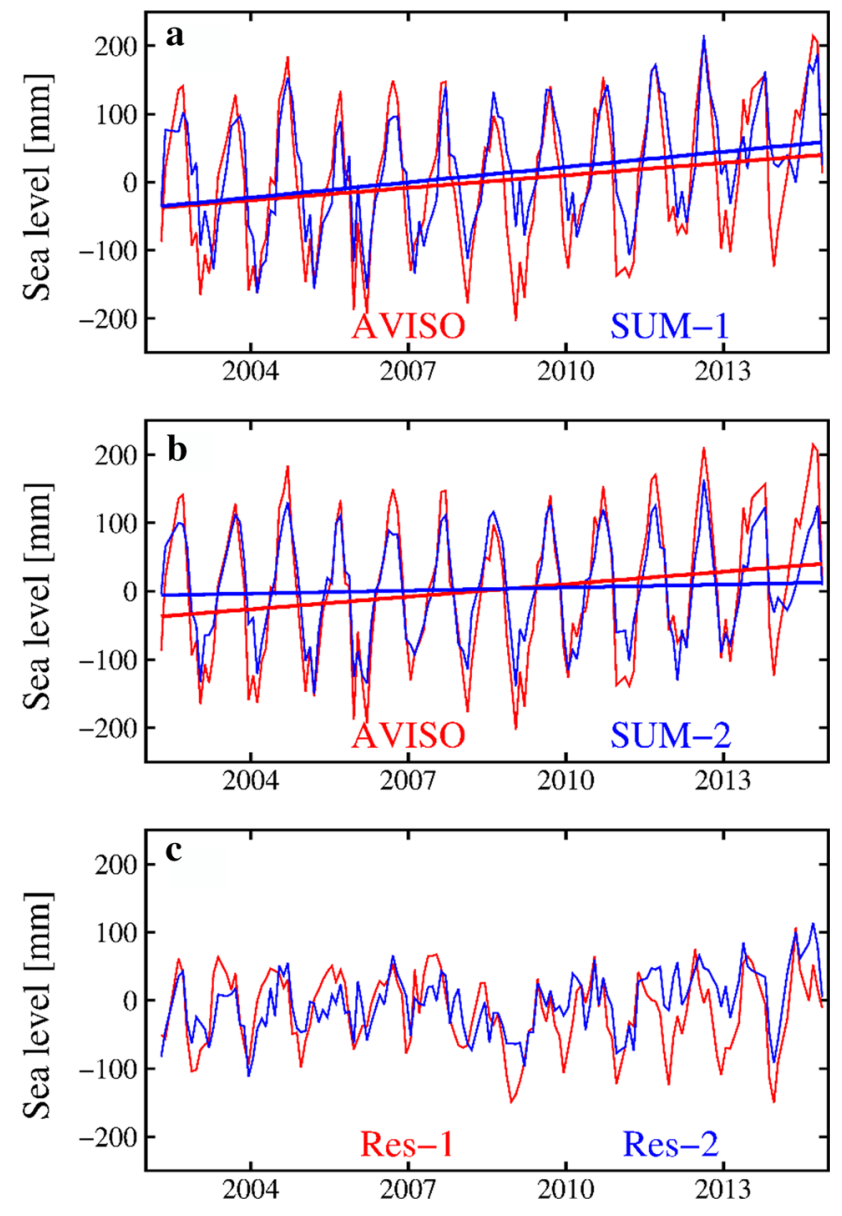

Fig. 7 Sea level change in the Bohai Sea. a The red curve is for AVISO, and the straight red line represents its linear trend; the blue curve is derived from the combination of JPL-M CRI and EN4, and the straight blue line represents its linear trend; $\mathbf{b}$ the red curve is for AVISO, and the straight red line represents its linear trend; the blue curve is derived from the combination of CSR-M and EN4, and the straight blue line represents its linear trend; c Res-1 indicates the difference between AVISO and SUM-1, and Res- 2 indicates the difference between AVISO and SUM-2

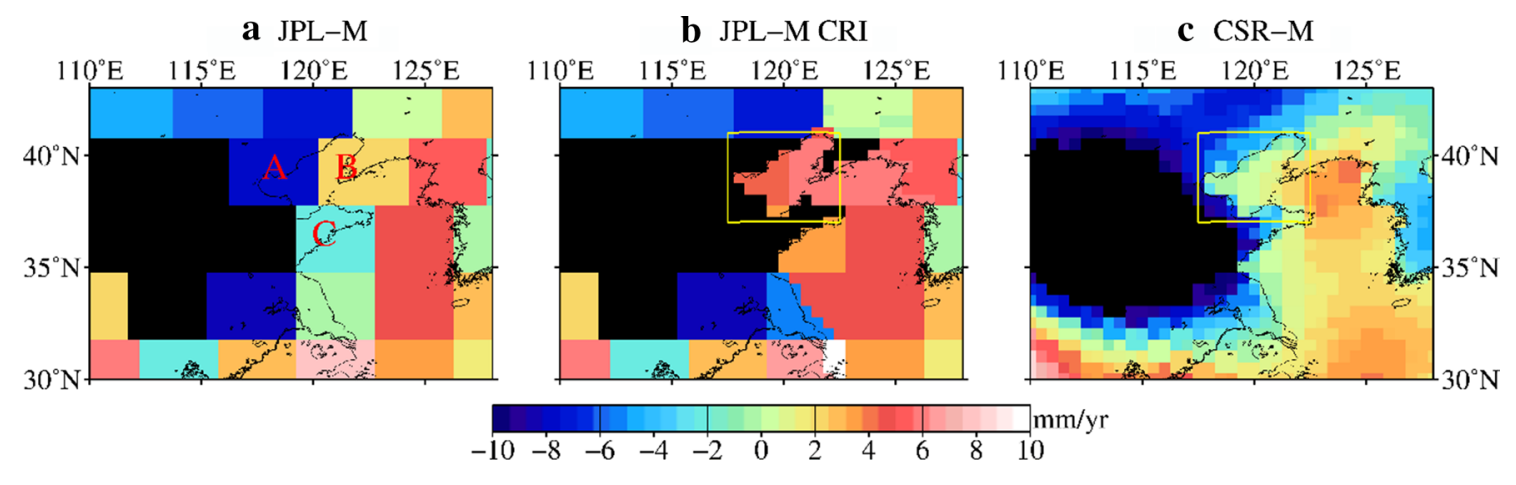

Fig. 6 Trend patterns from GRACE mascon. a JPL mascon (JPL-M) without leakage correction, A, B, and c mark three blocks; b JPL mascon with leakage correction, i.e., CRI algorithm; c CSR mascon (CSR-M) 
that CSR mascon approach requires further improvement, at least for regional scales. We also find that the rate of raw JPL-M (i.e., the block B) is only about $2.34 \mathrm{~mm}$ /year, which is larger than the CSR-M rate (Table 2). Given the principle of CRI algorithm (it takes information from the adjacent mascon to correct the target mascon), the corrected rate (Fig. 6b) is likely to be a coincidence. Indeed, the rate of the blocks near to the Bohai Sea is in agreement with the inferred rate (Table 2).

\section{Possible effect of sediment accumulation}

The sediment issue requires some comment. A 10-year model simulation (Bian et al. 2013) suggested a highly uneven spatial pattern of sediment accumulation, with small rates (1-2 mm/year) over the middle region of the Bohai Sea and significant rates $(>10 \mathrm{~mm} /$ year $)$ at the Yellow River estuary. Bian et al. (2013) also argued that only little of the sediment can be transported out of the Bohai Sea. However, our results do not support the detection of the sediment accumulation (including both trends and seasonal cycles) in the Bohai Sea by GRACE estimates. Nevertheless, sediment accumulation does occurs at local scale, e.g., the Yellow River estuary, implying that GRACE cannot detect such a local signal because of coarse resolution.

The evident mass increase in the Yellow Sea represents a major challenge for interpreting GRACE signals (Fig. 3a and Fig. 6), because satellite altimetry does not show such overwhelming positive trends. The sediment accumulation in the East China Sea has been studied. For instance, using GRACE estimates for the period 2002-2015, Liu et al. (2016) derived a $6 \mathrm{~mm} /$ year rate of sediment accumulation for the East China Sea inner shelf. They found that the magnitudes and spatial patterns of sediment derived from GRACE are in agreement with those from sediment-core measurements. Furthermore, a recent study by Chang et al. (2019), who employed an inversion of the sediment accumulation with multisource, also reported sediment trends along with seasonal cycles in the East China Sea. They explained the sediment seasonal cycles with ocean currents, which may result in the redistribution of sediment between the East China Sea and the Yellow Sea. However, the mass increase in the Yellow Sea has not been explored yet with GRACE estimates. A bold proposal is that significant amounts of sediment from the Yellow River is carried out of the Bohai Sea and deposited into the Yellow Sea. Note that this proposal is indeed in contrast to model results, which suggested that most of the sediment is accumulated in the interior of the Bohai Sea (Bian et al. 2013). To confirm this conjecture, more efforts (including advanced approaches and in situ measurements) should be made in future, e.g., the GRACE follow-on mission may provide a unique opportunity.

\section{Summary}

The time-variable gravity from GRACE mission had revealed many events that were characterized with broad spatial scales (e.g., water mass increase in the northwestern Pacific reported by Cheng et al. 2013b), enormous amount (e.g., mass loss in Greenland, see Chen et al. 2013 and $\mathrm{Mu}$ et al. 2017), or extreme increase in a small region $(\sim 38 \mathrm{~m}$ water level increase in an area of $\sim 400 \mathrm{~km}^{2}$, see Yi et al. 2017). Altimeter measurements, which are corrected for the steric change, show that the Bohai Sea is experiencing mass increase. Yet this increase is significantly attenuated in GRACE traditional SHCs products due to both serious leakage-in effect from the North China Plain and signal attenuation caused by smoothing. Hence, it is difficult to directly identify this event with GRACE SHCs. We examine the mascon solutions that initiate this study and find the increase in the Bohai Sea. We implement a reconstruction with multisource data to reproduce the signals observed by GRACE SHCs. Our reconstructed trend and the GRACE SHCs trends are both small, while the uncertainty is estimated to be large. The cause for the GRACE trend therefore remains ambiguous at this point. Nevertheless, our reconstruction demonstrates that the annual cycles of mass changes are mainly attributed to water mass variations instead of sediment changes.

Furthermore, an important implication of our results is that mascon solutions suggest better variability. For instance, compared with SHCs amplitudes, two mascon amplitudes are enhanced (see Tables 1,2) over the Baohai Sea. Globally, however, ocean mass variations from raw SHCs (i.e., neither filter nor destriping is applied to the SHCs) are in good agreement with those from mascon (see Chen et al. 2018; Uebbing et al. 2019). This hints that mascon solutions may be useful for investigating regional mass variations, because the GRACE SHCs suffer from stripes that are featured with shapes and sizes that both depend on locations. It has been argued that destriping algorithms remove the stripes along with the signals (Watkins et al. 2015). We employ the destriping algorithms (e.g., Swenson and Wahr 2006) and find no improvement in the Bohai Sea mass changes. However, community should be more careful with GRACE estimates when they aim at investigating variations in small regions that face complex geophysical environments.

Acknowledgements We thank Brian Gunter (editor), Jennifer Bonin (reviewer), and two anonymous reviewers who significantly improved the quality of this paper. This research was funded by the National Natural Science Foundation of China (Grant Nos. 41904081, 41731069, 41874032 and 41574013) and the National Key Research and Development Program of China (2016YFB0501701). Dapeng Mu acknowledged the financial support from China Postdoctoral Science Foundation (2018M640628) and State Key Laboratory of Geodesy and Earth's Dynamics (SKLGED2019-2-4-E). 
Author contributions DM conceived the idea and performed the data analysis; DM and TX wrote the manuscript; DM, TX, and GX discussed all the results.

Data availability The GRACE data can be downloaded from https:// grace.jpl.nasa.gov. The AVISO data are available at https://www.aviso .altimetry.fr. The EN4 data can be downloaded from https://www.metof fice.gov.uk/hadobs/en4/. Questions and requests for materials can be addressed to mdp321@126.com or thxu@sdu.edu.cn.

\section{Compliance with ethical standards}

Conflict of interest The authors declare no conflict of interest.

Open Access This article is licensed under a Creative Commons Attribution 4.0 International License, which permits use, sharing, adaptation, distribution and reproduction in any medium or format, as long as you give appropriate credit to the original author(s) and the source, provide a link to the Creative Commons licence, and indicate if changes were made. The images or other third party material in this article are included in the article's Creative Commons licence, unless indicated otherwise in a credit line to the material. If material is not included in the article's Creative Commons licence and your intended use is not permitted by statutory regulation or exceeds the permitted use, you will need to obtain permission directly from the copyright holder. To view a copy of this licence, visit http://creativecommons.org/licenses/by/4.0/.

\section{References}

A G, Wahr J, Zhong S (2013) Computations of the viscoelastic response of a 3-D compressible Earth to surface loading: an application to glacial isostatic adjustment in Antarctica and Canada. Geophys J Int 192:557-572

AVISO (2014) SSALTO/DUACS user handbook: (M)SLA and (M) ADT near-real time and delayed time products CLS-DOSNT-06-034, 4.1

Bi C, Bao X, Wan K (2015) The effect of decadal salinity variations on circulation in the Bohai Sea. Period Ocean Univ China (in Chinese) $45: 1-8$

Bian C, Jiang W, Greatbatch RJ (2013) An exploratory model study of sediment transport sources and deposits in the Bohai Sea, Yellow Sea, and East China Sea. J Geophys Res Oceans 118:5908-5923

Carton JA, Penny SG, Kalnay E (2019) Temperature and salinity variability in the SODA3, ECCO4r3, and ORAS5 ocean reanalyses, 1993-2015. J Clim 32:2277-2293

Cazenave A, Dominh K, Guinehut S et al (2009) Sea level budget over 2003-2008: a reevaluation from GRACE space gravimetry, satellite altimetry and Argo. Glob Planet Change 65:83-88

Cazenave A et al (2018) Global sea level budget 1993-present. Earth Syst Sci Data 10:1551-1590

Chambers DP, Wahr J, Nerem RS (2004) Preliminary observations of global ocean mass variations with GRACE. Geophys Res Lett 31:L13310. https://doi.org/10.1029/2004g1020461

Chambers DP, Wahr J, Tamisiea ME, Nerem RS (2010) Ocean mass from GRACE and glacial isostatic adjustment. J Geophys Res 115:B11415. https://doi.org/10.1029/2010jb007530

Chambers DP, Cazenave A, Champollion N, Dieng H, Llovel W, Forsberg R, von Schuckmann K, Wada Y (2017) Evaluation of the global mean sea level budget between 1993and 2014. Surv Geophys 38:309-327
Chang L, Tang H, Yi S, Sun W (2019) The trend and seasonal change of sediment in the East China Sea detected by GRACE. Geophys Res Lett 46:1250-1258

Chen JL, Wilson CR, Tapley BD (2013) Contribution of ice sheet and mountain glacier melt to recent sea level rise. Nat Geosci 6:549-552

Chen J, Tapley B, Save H, Tamisiea ME, Bettadpur S, Ries J (2018) Quantification of ocean mass change using Gravity Recovery and Climate Experiment, satellite altimeter, and Argo floats observations. J Geophys Res Solid Earth 123:10212-10225

Cheng MK, Tapley BD, Ries JC (2013a) Deceleration in the Earth's oblateness. J Geophys Res Solid Earth 118:740-747

Cheng X, Li L, Du Y, Wang J, Huang R-X (2013b) Mass-induced sea level change in the northwestern Pacific and its contribution to total sea level change. Geophys Res Lett 40:3975-3980

Dieng HB, Cazenave A, Meyssignac B et al (2017) New estimate of the current rate of sea level rise from a sea level budget approach. Geophys Res Lett 44:3744-3751

Farrell W, Clark J (1976) On postglacial sea-level. Geophys J R Astron Soc 46:647-667

Feng W, Lemoine JM, Zhong M et al (2014) Mass-induced sea level variations in the Red Sea from GRACE, steric-corrected altimetry, in situ bottom pressure records, and hydrographic observations. J Geodyn 78:1-7

Feng W, Shum CK, Zhong M, Pan Y (2018) Groundwater storage changes in China from satellite gravity: an overview. Remote Sens 10:674. https://doi.org/10.3390/rs10050674

Flechtner F, Dobslaw H, Fagiolini E (2014) AOD1B product description document for product release 05, Rev.4.2, 20 May 2014

Good SA, Martin MJ, Rayner NA (2013) EN4: quality controlled ocean temperature and salinity profiles and monthly objective analyses with uncertainty estimates. J Geophys Res Oceans 118:6704-6716

Guo J, Mu D, Liu X, Yan H, Dai H (2014) Equivalent water height extracted from GRACE gravity field model with robust independent component analysis. Acta Geophys 62:953-972

Johnson GC, Chambers DP (2013) Ocean bottom pressure seasonal cycles and decadal trends from GRACE Release-05: ocean circulation implications. J Geophys Res Oceans 118:1-13

Kusche J, Uebbing B, Rietbroek R, Shum CK, Khan ZH (2016) Sea level budget in the Bay of Bengal (2002-2014) from GRACE and altimetry. J Geophys Res Oceans 121:1194-1217

Liu Y-C, Hwang C, Han J, Kao R, Wu C-R, Shih H-C, Tangdamrongsub N (2016) Sediment-mass accumulation rate and variability in the East China Sea detected by GRACE. Remote Sens 8:777. https ://doi.org/10.3390/rs8090777

Llovel W, Lee T (2015) Importance and origin of halosteric contribution to sea level change in the southeast Indian Ocean during 2005-2013. Geophys Res Lett 42:1148-1157

Mouyen M, Longuevergne L, Steer P, Crave A, Lemoine J-M, Save H, Robin C (2018) Assessing modern river sediment discharge to the ocean using satellite gravimetry. Nat Commun 9:3384. https://doi. org/10.1038/s41467-018-05921-y

Mu D, Yan H, Feng W, Peng P (2017) GRACE leakage error correction with regularization technique: case studies in Greenland and Antarctica. Geophys J Int 208:1775-1786

Peltier WR, Argus DF, Drummond R (2017) Comment on "An assessment of the ICE-6G_C (VM5a) glacial isostatic adjustment model" by Purcell et al. J Geophys Res Solid Earth 123:2019-2028

Reager JT, Gardner AS, Famiglietti JS, Wiese DN, Eicker A, Lo M-H (2016) A decade of sea level rise slowed by climate-driven hydrology. Science 351:699-703

Rietbroek R, Brunnabend SE, Kusche J et al (2016) Revisiting the contemporary sea-level budget on global and regional scales. Proc Natl Acad Sci USA 2016(113):1504-1509 
Rowlands DD, Luthcke SB, McCarthy JJ et al (2010) Global mass flux solutions from GRACE: a comparison of parameter estimation strategies-mass concentrations versus Stokes coefficients. J Geophys Res 115:B01403. https://doi.org/10.1029/2009jb006546

Save H, Bettadpur S, Tapley BD (2016) High-resolution CSR GRACE RL05mascons. J Geophys Res Solid Earth 121:7547-7569

Spada G (2017) Glacial isostatic adjustment and contemporary sea level rise: an overview. Surv Geophys 38:153-185

Stammer D, Cazenave A, Ponte RM, Tamisiea ME (2013) Causes for contemporary regional sea level changes. Annu Rev Mar Sci 5:21-46

Swenson S, Wahr J (2006) Post-processing removal of correlated errors in GRACE data. Geophys Res Lett 33:L08402. https://doi. org/10.1029/2005GL025285

Swenson S, Chambers D, Wahr J (2008) Estimating geocenter variations from a combination of GRACE and ocean model output. J Geophys Res 113:B08410. https://doi.org/10.1029/2007jb005338

Tamisiea ME (2011) Ongoing glacial isostatic contributions to observations of sea level change. Geophys J Int 186:1036-1044

Tapley BD et al (2019) Contributions of GRACE to understanding climate change. Nat Clim Change 9:358-369

Uebbing B, Kusche J, Rietbroek R, Landerer FW (2019) Processing choices affect ocean mass estimates from GRACE. J Geophys Res Oceans 124:1029-1044

Wahr J, Molenaar M, Bryan F (1998) Time variability of the Earth's gravity field: hydrological and oceanic effects and their possible detection using GRACE. J Geophys Res 103:30205-30229
Wahr J, Smeed DA, Leuliette E, Swenson S (2014) Seasonal variability of the Red Sea, from satellite gravity, radar altimetry, and in situ observations. J Geophys Res Oceans 119:5091-5104

Watkins MM, Wiese DN, Yuan DN et al (2015) Improved methods for observing Earth's time variable mass distribution with GRACE using spherical cap mascons. J Geophys Res Solid Earth 120:2649-2671

Wiese DN, Landerer FW, Watkins MM (2016) Quantifying and reducing leakage errors in the JPL RL05M GRACE mascon solution. Water Resour Res 52:7490-7502

Yan H, Chen W, Yuan L (2016) Crustal vertical deformation response to different spatial scales of GRACE and GCMs surface loading. Geophys J Int 204:505-516

Yang ZS, Liu JP (2007) A unique Yellow River-derived distal subaqueous delta in the Yellow Sea. Mar Geol 240:169-176

Yi S, Sun W, Heki K, Qian A (2015) An increase in the rate of global mean sea level rise since 2010. Geophys Res Lett 42:3998-4006

Yi S, Song C, Wang Q, Wang L, Heki K, Sun W (2017) The potential of GRACE gravimetry to detect the heavy rainfall-induced impoundment of a small reservoir in the upper Yellow River. Water Resour Res 53:6562-6578

Zuo H, Balmaseda M, Tietsche S, Mogensen K, Mayer M (2019) The ECMWF operational ensemble reanalysis-analysis system for ocean and sea-ice: a description of the system and assessment. Ocean Sci Discuss. https://doi.org/10.5194/os-2018-154 